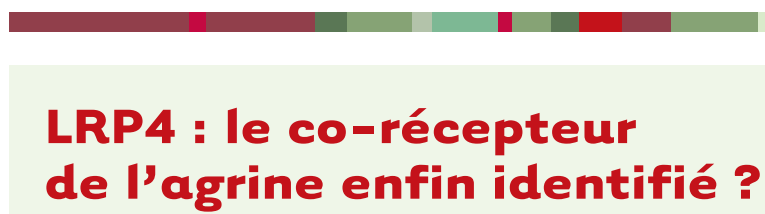

Jean Cartaud, Annie Cartaud
Laboratoire

de Biologie Cellulaire des Membranes,

Institut Jacques Monod, UMR 7592,

CNRS, Université Paris 7,

2, place Jussieu,

75251 Paris Cedex 05, France.

cartaud@ijm.jussieu.fr

mutants de MuSK, ces souris ont des anomalies de la différenciation pré- et post-synaptique. Lrp4 est de plus préférentiellement transcrit dans les noyaux sous neuraux de la fibre musculaire, comme le sont les gènes codant pour les protéines post-synaptiques en général. Ces observations ont conduit les groupes des Professeurs Steven Burden, de la New York University Medical School [6] et Lin Mei, du Medical College of Georgia [7] à tester I'hypothèse que Lrp4 soit impliqué dans la signalisation agrineMuSK. Les résultats publiés par ces deux groupes sont convergents et démontrent que Lrp4 est un co-récepteur de l'agrine, qu'il forme un complexe avec MuSK et qu'il est nécessaire à la signalisation de MuSK, conduisant au recrutement du RACh à la JNM.

Lrp4 contient un domaine extracellulaire amino-terminal composé de multiples répétitions de motifs EGF (epidermal growth factor) et de motifs RLDL, un domaine transmembranaire et une courte région carboxy-terminale sans aucun motif catalytique identifiable. Dans les fibres musculaires où il est spécifiquement exprimé, Lrp4 est co-localisé à la JNM avec le RACh et MuSK. Dans les lignées de cellules musculaires issues de souris mutantes pour Lrp4, l'agrine n'est plus capable d'activer MuSK, ceci montrant que Lrp4 est nécessaire à la stimulation de la phosphorylation de MuSK. Les deux groupes ont mis en évidence que le domaine extracellulaire de Lrp4 lie sélectivement et à haute affinité les isoformes d'agrine nerveuse. Dans des cellules non musculaires (BaF3 ou HEK) co-exprimant Lrp4 et MuSK, des expériences d'immuno-précipitation 
montrent que l'agrine stimule la phosphorylation de MuSK et augmente leur interaction. Ces dernières observations suggèrent que Lrp4 et MuSK forment un complexe même en l'absence du ligand, et que la liaison de l'agrine à Lrp4 potentialise l'interaction (Figure 1). Enfin, la suppression de l'expression de Lrp4 dans les myotubes $\mathrm{C} 2 \mathrm{C} 12$ diminue à la fois la liaison de l'agrine nerveuse, l'activation de MuSK par l'agrine, et la formation d'agrégats de RACh. En conclusion, l'ensemble de ces observations indique que Lrp4 est un co-récepteur de l'agrine nécessaire à la signalisation de MuSK et au recrutement du RACh.

\section{Nouvelles perspectives}

Les résultats de ces deux études sont importants à plusieurs titres; non seulement ils confirment l'existence d'un co-récepteur de l'agrine nécessaire à la différenciation synaptique, mais ils rendent compte d'un certain nombre d'observations jusqu'ici difficiles à interpréter. En effet, durant le développement, quand l'axone moteur entre en contact avec la fibre musculaire, MuSK est activé indépendamment de l'agrine dans la future zone neurale où les premiers agrégats de RACh apparaissent [9]. Ce «prépatterning » est sensible à la quantité de MuSK dans la fibre ainsi qu'à Lrp4. L'auto-association de Lrp4 et de MuSK, qui conduit à leur trans-phosphorylation et à l'activation de MuSK en l'absence de ligand explique pourquoi la présence de l'agrine n'est pas requise dans les premières étapes de recrutement du RACh.

L'identification de Lrp4 apporte aussi des clés pour comprendre le mécanisme par lequel l'agrine agrège les RACh. Lrp4 peut en effet réguler l'activité de MuSK en favorisant son auto-dimérisation. Lrp4 pourrait aussi, via son motif NPXY juxta-membranaire, être impliqué dans le recrutement d'adaptateurs cytoplasmiques contenant un domaine PTB (phosphotyrosine binding) tel DOK-7, essentiel à la différenciation synaptique [10].

Enfin, la découverte de Lrp4 soulève la possibilité d'une participation de la voie Wnt dans la formation de la JNM. Les lipoprotéines Wnt sont des molécules de signalisation sécrétées qui sont impliquées dans l'embryogenèse, en particulier dans la formation de la JNM chez la drosophile et le vers C. elegans. Les protéines Wnt lient les récepteurs Frizzled et Lrp5/6, puis mettent en jeu la protéine adaptatrice Dishevelled (Dvl) [11] qui interagit avec Frizzled pour déclencher la signalisation. De plus, plusieurs molécules de signalisation de la cascade Wnt, telles que APC (adenomatous polyposis coli) et $\beta$-caténine sont aussi, ainsi que Dvl, partenaires de la voie de signalisation de MuSK. Ainsi, bien qu'il ne soit pas établi que la voie Wnt soit directement impliquée dans la différenciation synaptique chez les mammifères et bien que Lrp4 ne lie pas Wnt-1, il demeure possible que la voie de signalisation Agrine-Lrp4-MuSK soit régulée par un ligand de la voie Wnt qui pourrait interagir avec Lrp4 ou MuSK (qui contient un motif Frizzled). Ces questions ouvrent de nouvelles voies de recherches non seulement dans le mécanisme de recrutement du RACh à la JNM, mais aussi dans le cadre des synapses du système nerveux central. $\diamond$

LRP4: the coreceptor

for Agrin identified at last?

\section{RÉFÉRENCES}

1. Sanes JR, Lichtman JW. Induction, assembly, maturation and maintenance of a postsynaptic apparatus. Nat Rev Neurosci 2001 ; 2 : 791-805.

2. Gautam M, Noakes PG, Moscoso L, et al. Defective neuromuscular synaptogenesis in agrin-deficient mutant mice. Cell 1996; $85: 525-35$.

3. Glass DJ, Bowen DC, Stitt TN, et al. Agrin acts via a MuSK receptor complex. Cell 1996; 85 : 513-23.

4. Engel AG, Shen XM, Selcen D, Sine SM. Further observations in congenital myasthenic syndromes. Ann Ny Acad Sci 2008 ; 1132 : 104-13.

5. Strochlic L, Cartaud A, Cartaud J. The synaptic musclespecific kinase (MuSK) complex: new partners, new functions. Bioessays 2005; 27 : 1129-35.

6. Kim N, Stiegler AL, Cameron TO, et al. Lrp4 is a receptor for agrin and forms a complex with MuSK. Cell 2008; $135: 334-42$.

7. Zhang B, Luo S, Wang $Q$, et al. LRP4 serves as a coreceptor of agrin. Neuron $2008 ; 60: 285-97$.

8. Weatherbee SD, Anderson KV, Niswander LA. LDLreceptor related protein 4 is crucial for formation of the neuromuscular junction. Development 2006 ; 133 : 4993-5000.

9. Kummer TT, Misgeld T, Sanes JR. Assembly of the posysynaptic membrane at the neuromuscular junction: paradigm lost. Curr Opin Neurobiol 2006 ; $16: 74-82$.

10. Okada K, Inoue A, Okada M, et al. The muscle protein DOK-7 is essential for neuromuscular synaptogenesis. Science 2006 ; 312 : 1802-5.

11. Luo Z, Wang $Q$, Zhou J, et al. Regulation of AChR clustering by dishevelled interacting with MuSK and PAKl. Neuron 2002 ; 35 : 489-505.

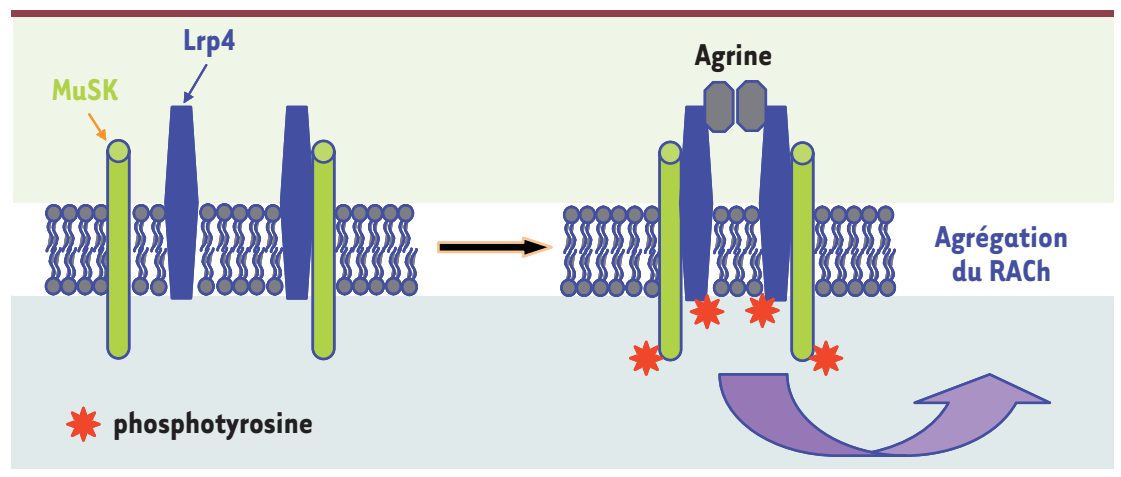

Figure 1. Modèle illustrant les interactions entre Lrp4, MuSK et agrine. En l'absence d'agrine, MuSK et Lrp4 sont capables de s'associer. L'interaction est accrue par l'agrine, ce qui entraîne leur trans-phosphorylation (*). Une fois phosphorylé, MuSK active une cascade de signalisation [5] conduisant au recrutement des RACh et à la différenciation synaptique. La représentation dimérique de MuSK est hypothétique, une oligomérisation du complexe est aussi possible (modifié d'après $[6,7]$ ). 